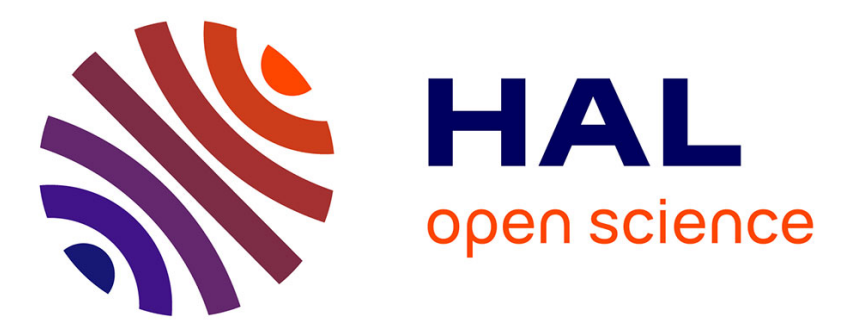

\title{
SSVEP enhancement based on Canonical Correlation Analysis to improve BCI performances
}

Emmanuel Kalunga, Karim Djouani, Yskandar Hamam, Sylvain Chevallier, Eric Monacelli

\section{- To cite this version:}

Emmanuel Kalunga, Karim Djouani, Yskandar Hamam, Sylvain Chevallier, Eric Monacelli. SSVEP enhancement based on Canonical Correlation Analysis to improve BCI performances. AFRICON 2013, Sep 2013, Pointe-Aux-Piments, Mauritius. 10.1109/AFRCON.2013.6757776 . hal-01352059

\section{HAL Id: hal-01352059 https://hal.science/hal-01352059}

Submitted on 5 Aug 2016

HAL is a multi-disciplinary open access archive for the deposit and dissemination of scientific research documents, whether they are published or not. The documents may come from teaching and research institutions in France or abroad, or from public or private research centers.
L'archive ouverte pluridisciplinaire HAL, est destinée au dépôt et à la diffusion de documents scientifiques de niveau recherche, publiés ou non, émanant des établissements d'enseignement et de recherche français ou étrangers, des laboratoires publics ou privés. 


\title{
SSVEP Enhancement Based on Canonical Correlation Analysis to Improve BCI Performances
}

\author{
Emmanuel Kalunga*†, Karim Djouani*, Yskandar Hamam*, Sylvain Chevallier ${ }^{\dagger}$ and Eric Monacelli ${ }^{\dagger}$ \\ *Department of Electrical Engineering/French South African Institute of Technology \\ Tshwane University of Technology, Pretoria 0001, South Africa \\ Email: emmanuelkalunga.k@gmail.com \\ ${ }^{\dagger}$ Laboratoire d'Ingénierie des Systèmes de Versailles \\ Université de Versailles Saint-Quentin, Velizy 78140, France
}

\begin{abstract}
Brain Computer Interfaces (BCI) rely on brain waves signal, such as electro-encephalogram (EEG) recording, to endow a disabled user with non-muscular communication. Given the very low signal-to-noise ratio of EEG, a signal enhancement phase is crucial for ensuring decent performances in BCI systems. Several methods have been proposed for EEG signal enhancement, such as Independent Component Analysis, Common Spatial Pattern, and Principal Component Analysis. We show that Canonical Correlation Analysis (CCA), initially introduced to SSVEP-based BCI as a feature extraction method, is a good candidate for such preprocessing state. Evaluation is performed on a recording from 5 subjects during a BCI task based on Steady-State Visual Evoked Potentials (SSVEP). We demonstrate that CCA significantly improves classification performances in SSVEP-based BCIs.
\end{abstract}

\section{INTRODUCTION}

Brain Computer Interface (BCI) systems provide an interface for communicating and controlling a physical environment using brain signals and bypassing the normal neuromuscular pathways [1]. It thus constitutes an alternative means of control for the large population of people with limited to non-existent muscular abilities. In general, BCI can be used to improve human performances not achievable otherwise due to limited physical capabilities. Development in EEG measurement techniques and opportunities seen in BCI have been attracting research interest for the last three decades.

To ensure reliability of BCI systems, robust signal processing techniques are needed for accurate and fast classification of users' intentions. However challenges due the nature of EEG, i.e. poor signal-to-noise ratio, poor topographical resolution, make it difficult for signal processing algorithms to accurately detect users' intentions. Indeed a common approach is to preprocess EEG recording with signal enhancement techniques [2], [3]. This contribution focus on the application of Canonical Correlation Analysis (CCA) to enhance the induced brain responses to specific stimuli, known as Steady-State Visual Evoked Potentials (SSVEP) prior to feature extraction and classification.

SSVEP is the response to a sequence of frequent visual stimuli [4]. Neuronal activities in the primary visual cortex are synchronised with the stimulation frequencies, including its harmonics. These frequencies are expected to be observable in the EEG measured from the primary visual cortex. Hence, enhancing SSVEP signal is, in other words, increasing the visibility of the SSVEP characteristics, which are mainly the stimuli frequencies.

Signal enhancement methods are performed on the EEG to increase the signal-to-noise ratio. This is the most challenging part of the EEG analysis. In fact, various noises that interfere with EEG contribute to its poor signal-to-noise ratio. The common noises that overlap with the EEG signal are (1) the electrooculogram (eye-blinking and eye movement), (2) the electrocardiogram (heartbeat), (3) electro-myogram (muscle movements) and (4) ongoing brain activities. These noise sources are all generated by the subject himself. EEG is also affected by environmental noises such as the $50 / 60 \mathrm{~Hz}$ component from the AC power lines. Methods such as Independent Component Analysis (ICA) [5], Common Spatial Pattern (CSP) [6], and Principal Component Analysis (PCA) [7], [8] have been used for EEG signal enhancement.

This contribution describes the application of CCA for signal enhancement. It is a method of correlating linear relationships between two multidimensional variables[9]. It was initially proposed by Hotelling in 1936. For two sets of multidimensional variables, CCA aims at finding a new basis such that the two sets of variables have maximum correlation when projected on this subspace. CCA was introduced in SSVEP signal processing only in 2007, by Zhonglin Lin et al. [10] where it was used to analyse the relationship between the stimulus signals and EEG signals from multiple channels in a local area. For each EEG sample, the correlation coefficients are computed using CCA, these coefficients are used as features for classification. Following this work, several studies have also investigated CCA for SSVEP-based BCI as a feature extraction method [11], [12], [13].

The proposed approach is to investigate CCA as a signal enhancement method and not as a feature extraction method. We make use of the ability of CCA to handle multichannel EEG and find the space in which EEG samples correlate the most with the stimuli. To enhance SSVEP features, EEG samples are projected into that space. Power spectral density analysis (PSDA) is then used for feature extraction. To evaluate the impact that CCA based signal enhancement has on classification performances, an SVM classifier is used. The CCA weights are computed once during the training phase, and during the evaluation phase, the signal enhancement process consists of a simple matrix multiplication. Unlike methods like the ICA, the CCA yields effective weights (spacial filters) with relatively small training sets. 
Section II presents the material and methods used for EEG recording, including the experimental paradigm and the CCA approach for signal enhancement. Section III details the results and discusses the impact of the CCA-based signal enhancement on the classification performance through a comparison with ICA-based signal enhancement, and a classification method that is not preceded by any signal enhancement technique.

\section{METHODS}

\section{A. Material}

Eight high-purity gold crown butterfly active electrodes (g.BUTTERFLY), mounted on a G.GAMMACAP, are used for EEG measurement. The EEG signal are sampled at a rate of $256 \mathrm{~Hz}$. Openvibe [14] is used to acquire the signal.

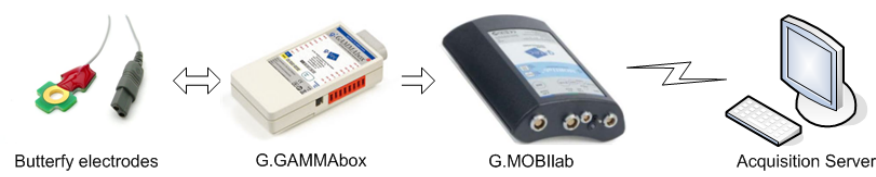

Fig. 1. Acquisition material: The EEG are recorded with electrodes, the signal is amplified and sent to a computer running OpenVIBE.

The EEG recorded during experiments are later analysed using MATLAB 7. For SSVEP stimulation, flash stimulus technique have been chosen. Computer monitors can only flash at multiple frequencies of its refresh rate. To avoid this limitation, light emitting diodes (LED) are used to flash stimuli at $13 \mathrm{~Hz}, 17 \mathrm{~Hz}$, and $21 \mathrm{~Hz}$. The frequencies are generated by a PIC 18F2550.

\section{B. Subjects and EEG Recording}

Five healthy subjects (aged between 22 and 30) participated in the offline experiment. Four subjects did not have any previous experience with $\mathrm{BCI}$, and one had participated in other BCI (P300 and motor imagery) experiments and had a good understanding of the SSVEP principle. The 8 active electrodes were in mono-polar configuration placed according to the 10/20 system on Oz, O1, O2, POz, PO3, PO4, PO7, and PO8. The ground was placed on $\mathrm{Fz}$ and the reference was located on the right (or left) hear mastoid.

The experiments were done at the Laboratoire d'Ingénierie des Systèmes de Versailles (LISV) of the Université de Versailles Saint-Quentin-en-Yvelines. The subjects were seated comfortably in a chair facing the computer screen placed at about $60 \mathrm{~cm}$. Three LED arrays are placed on top, left and right sides of the computer screen and are the experimental targets.

In a recording session the subject was requested, in a random order, to look at blinking LED array, hereafter called stimuli. He was prompted to do so by a triangular cue appearing on the computer screen, on the left side to gaze at the left stimulus (i.e. $17 \mathrm{~Hz}$ ), on the right side to gaze at the right stimulus (i.e. $21 \mathrm{~Hz}$ ), on top to gaze at the top stimulus (i.e. 13 $\mathrm{Hz}$ ) and at the center to gaze at the center of the screen where no stimulus is flashed. All LEDs arrays are flashing for the

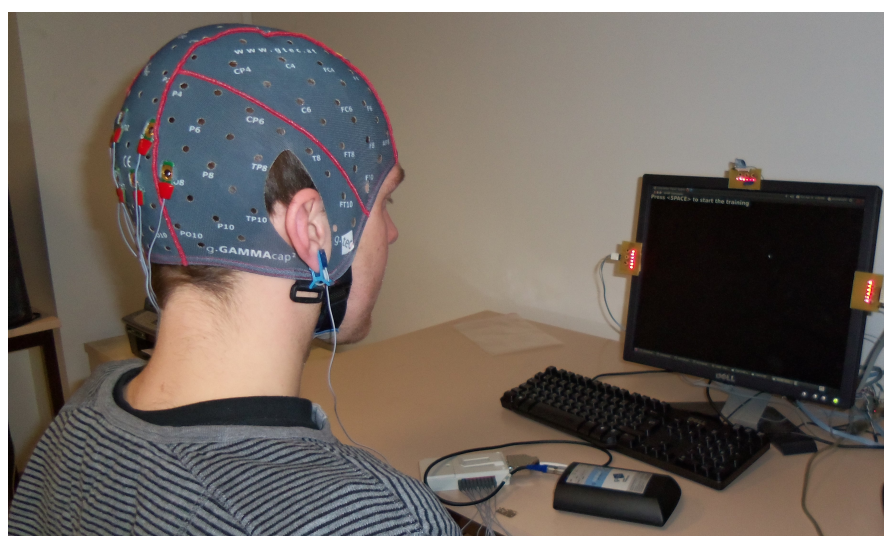

Fig. 2. Offline EEG recording. An array of 6 red LED's is used for each stimulus target. They are mounted on the sides of a 17 inches computer monitor which is used to display the experiment cues.

whole recording time. In a session, 10 trials were recorded per flash stimulus, for a total of 40 trials per session. During a trial the subject gazes at the flash stimuli for a period of 5 seconds followed by a 3 second break where the subject is supposed to look at center of the computer screen (no flash). The subject was advised not to blink nor move eyes while gazing at any of the three flash stimuli. The subject was allowed to blink the eyes during the trial looking at the center of the screen. After a session, the subject was given a 2-minutes break. Four sessions were recorded per subject and a total of 160 trials were recorded for each subject. Since the EEG were recorded for later analysis, no signal processing was done online and no feedback was provided to the subject.

\section{CCA Signal Enhancement}

Let $S_{x}$ and $S_{y}$ be the two sets of multidimensional variables taken from a multivariate random vector of the form $(x, y)$, with $S_{x}=\left(x_{1}, \ldots, x_{n}\right)$ and $S_{y}=\left(y_{1}, \ldots, y_{n}\right)$. CCA finds the direction $w_{x}$ and $w_{y}$ such that after projecting $x$ onto $w_{x}$ and $y$ onto $w_{y}$, two samples $\left(S_{x, w_{x}}\right.$ and $\left.S_{y, w_{y}}\right)$ with new coordinates are found and have a maximum correlation $\rho$.

$$
\begin{aligned}
\rho & =\max _{w_{x}, w_{y}} \operatorname{corr}\left(S_{x} w_{x}, S_{y} w_{y}\right) \\
& =\max _{w_{x}, w_{y}} \frac{\left\langle S_{x} w_{x}, S_{y} w_{y}\right\rangle}{\left\|S_{x} w_{x}\right\|\left\|S_{y} w_{y}\right\|}
\end{aligned}
$$

$\rho$ is rewritten as in [9]:

$$
\rho=\max _{w_{x}, w_{y}} \frac{w_{x}^{\prime} C_{x y} w_{y}}{\sqrt{w_{x}^{\prime} C_{x x} w_{x} w_{y}^{\prime} C_{y y} w_{y}}} .
$$

where $C_{x x}$ and $C_{y y}$ are the within-sets covariance matrices and $C_{x y}$ is the between-sets covariance matrix.

Solving Equation (1) is equivalent to maximizing the numerator subject to

$$
\begin{aligned}
& w_{x}^{\prime} C_{x x} w_{x}=1 \\
& w_{y}^{\prime} C_{y y} w_{y}=1
\end{aligned}
$$


This optimization problem could be solved with various techniques. Here we used the approached proposed by Hardoon et al. [9].

When applied to SSVEP, CCA finds the maximum correlation between $\mathrm{M}$ samples of EEG signal recorded from $\mathrm{N}$ channels, $X(N \times M)$ and the reference signal $Y(R \times M)$. $R$ is the number of variables in the reference signal. The reference signal and the EEG signal might be of different sizes but should have the same number of observed samples. The reference signal is generated as in [10]:

$$
Y=\left[\begin{array}{c}
\sin (2 \pi \times 1 \times f) \\
\cos (2 \pi \times 1 \times f) \\
\sin (2 \pi \times 2 \times f) \\
\cos (2 \pi \times 2 \times f) \\
\vdots \\
\sin (2 \pi \times m \times f) \\
\cos (2 \pi \times m \times f)
\end{array}\right]
$$

where $m$ is the number of harmonics used and $f$ is the fundamental frequency of the flash stimuli corresponding to the recorded EEG sample $X$.

The reference signals used for the $\mathrm{CCA}$ in the training contain the fundamental frequency of the trial stimulus and its harmonics. This allows CCA to investigate not only the fundamental stimulus frequency, but also the harmonics. Both cosine and sine are used. This alleviates the phase mismatch in CCA due to the fact that, like the standard correlation, CCA is phase locked and the recording techniques used in our experiments do not provide any synchronisation between the generation of flash stimuli and EEG recording.

In the training phase, CCA is applied separately to EEG samples from different classes. For $C$ classes (i.e. $C$ flash stimuli and $C$ reference signals $Y$ ), the process will result in $C$ projection vectors $w_{x_{C}}$. The one that yields the larger correlation with all EEG samples regardless of their class is selected.

CCA has been used in SSVEP studies as a feature extraction method where the classification decision was based on the correlation coefficients $\rho_{C}$. The class whose reference signal $Y$ yields the largest $\rho_{C}$ with the EEG sample is taken as the label of the sample [10]. In the proposed approach, CCA is used to enhance the SSVEP features of the EEG sample. Hence a feature extraction can be performed on the enhanced EEG signal and the extracted features fed into standard classifiers.

To evaluate the performance of the CCA based signal enhancement, we propose a comparison with an ICA-based signal enhancement method, a method commonly used for EEG enhancement in numerous BCI studies. The ICA algorithm implemented for this contribution is similar to the one described in [5].

\section{Feature Extraction and Classification}

The PSDs features are used to characterise the EEG samples. An estimate of power spectral density is obtained by performing a 1000-points fast Fourier transform on the enhanced signal. A Support Vector Machine (SVM) is chosen for feature classification. SVM are known for their robustness and possess good generalization properties, they are less prone to overfit and require only a few parameters to be tuned. SVM exhibits good EEG classification, though only a few studies have been investigated its application in SSVEP.

The training set is separated in $Q$ training groups with their corresponding labels. This yields $Q$ training groups of size $T \times F$ each, where $T$ is the number of trials per class, and $F$ is the dimension of the feature vector. Thus, feature vectors of class trials constitute samples of the training group of this class. The elements of feature vector $\mathbf{f}_{i}$ are normalised into $\mathbf{f}_{i_{n}}$ as:

$$
\mathbf{f}_{i_{n}}=\frac{\mathbf{f}_{i}}{\sum_{j=1}^{F} \mathbf{f}_{j}}
$$

To avoid the influence of outliers in the classification decision, $\nu$ samples (3 for the described experiment), the furthest from the centre of the group, are rejected from each training group. The training is carried out by LIBSVM as described in [15].

\section{E. Evaluation Method}

To avoid an evaluation of the classifier based on overfitted data and to take into consideration the inter trial and inter session variability, the performance measurements are computed in a cross-validation process. A 4-fold cross-validation is performed by dividing the entire EEG data set (training and test sets) into 4 partitions of 30 trials each. Each partition is then used once for testing while the remaining partitions are used for training.

\section{RESULTS AND DISCUSSION}

This section shows the impact that CCA based EEG enhancement has on SSVEP identification and classification. The performances of this method are compared to the ones of ICA based signal enhancement. Figure 3 illustrates a segment of 2 seconds of EEG signals from 6 channels, recorded during a $17 \mathrm{~Hz}$ trial. Filtering this multichannel EEG. with the CCA spatial filter obtained from the training phase, yields a signal with better SSVEP characteristics, shown in Figure 4. Before applying the CCA filter, it is not easy, from the frequency spectrum of Figure 3, to identify the SSVEP frequency. Only channels $\mathrm{O} 1$ and PO3 have the highest peak in their frequency spectrum at $17 \mathrm{~Hz}$. In the remaining channels, the highest peak is on other frequencies. The result of the CCA filtering plotted in Figure 4 proves that CCA can significantly enhance the SSVEP features. The $17 \mathrm{~Hz}$ component in the spectrum is clearly the largest power of the signal.

Figure 5 depicts a comparison of SVM classification performances, in terms of accuracy and information transfer rate (ITR), between (1) the SVM classification with no prior signal enhancement, (2) the SVM classification preceded with an ICA based signal enhancement, and (3) the SVM classification preceded with the proposed CCA based signal enhancement. The SSVEP trial length is varied from 0.5 to 5 seconds. In longer trials, subjects have got enough time to gaze at flash stimuli and demonstrate a good SSVEP response. The proposed CCA based signal enhancement method keeps the classification accuracy beyond $95 \%$ for trial from 2 to 5 seconds long. These results confirm that ICA signal enhancement improves SSVEP's classification performances, as demonstrated in [5] and [16]. The results also demonstrate that 

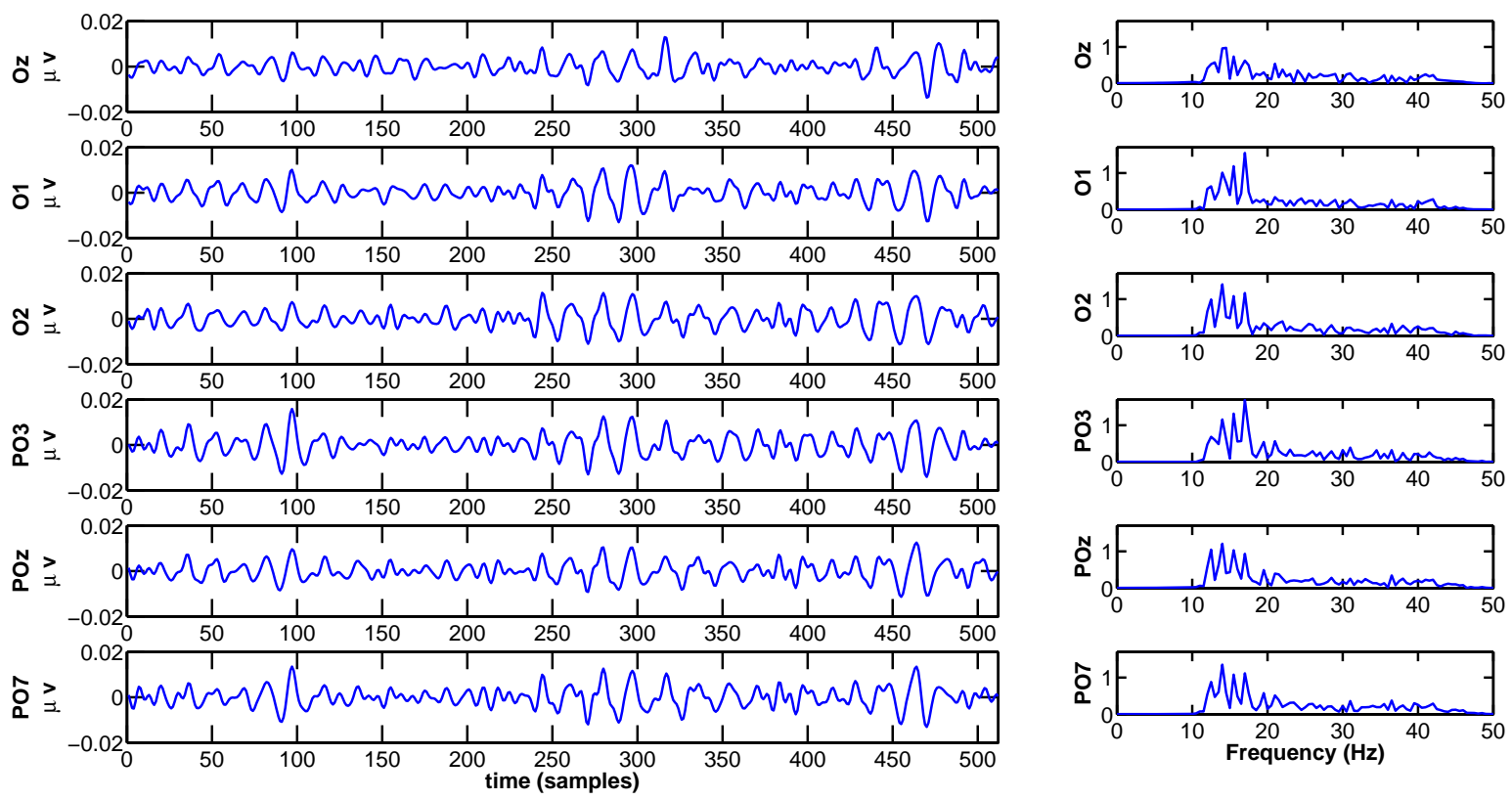

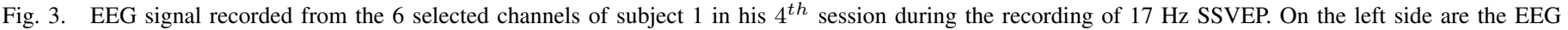
signals in the time domain and on the right side their frequency spectrum. For all channels the signal is bandpass filtered between $12 \mathrm{~Hz}$ and $45 \mathrm{~Hz}$.
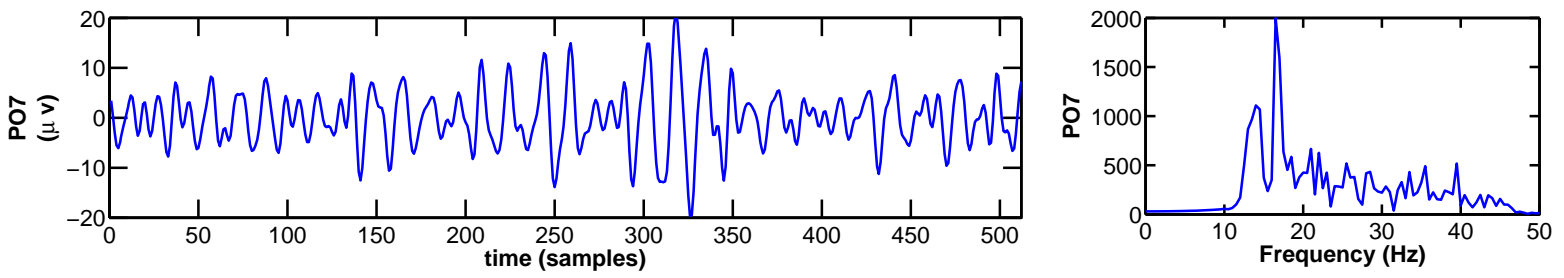

Fig. 4. CCA filtered EEG signal. On the left side is the signal in the time domain and on the right side the frequency spectrum.

the proposed CCA based signal enhancement further improves these performances, and yield better results for any trial length. Both ICA and CCA handle multichannel EEG signals, taking into account relevant SSVEP features that are scattered over different EEG channels. After obtaining the independent components (ICs), ICA still has to select the ICs that contains most of SSVEP features. In this endeavour, ICA rejects some ICs thus losing some of the relevant features (ICA does not completely separate the components from different sources). In CCA based signal enhancement, this challenge is not present, although, as a spacial filter, CCA might also loose some of the relevant features. The result of CCA signal enhancement is a single EEG signal with reduced dimensionality (from multivariate to univariate) and containing the relevant SSVEP features from all channels.

\section{CONCLUSION}

In this work, CCA has been used as a method for SSVEP signal enhancement. The method was applied in the signal processing of EEG data recorded from 5 subjects. To evaluate the impact of the method on EEG classification, PSD features extracted from the enhanced signal are classified using an SVM. The results demonstrate that using CCA for signal enhancement improves classification performances, and yields better results than using ICA.

\section{ACKNOWLEDGMENT}

The experiments conducted in France have been supported by the Foundation EADS through the project Cerebraptic.

\section{REFERENCES}

[1] J. R. Wolpaw, N. Birbaumer, D. J. McFarland, G. Pfurtscheller, and T. M. Vaughan, "Brain-computer interfaces for communication and control," Clinical Neurophysiology, vol. 113, no. 6, pp. 767-791, 2002.

[2] B. Blankertz, K.-R. R. Müller, G. Curio, T. M. Vaughan, G. Schalk, J. R. Wolpaw, A. Schlögl, C. Neuper, G. Pfurtscheller, T. Hinterberger, M. Schröder, and N. Birbaumer, "The BCI competition 2003: progress and perspectives in detection and discrimination of EEG single trials," Biomedical Engineering, IEEE Transactions on, vol. 51, no. 6, pp. 1044-1051, 2004.

[3] B. Blankertz, K.-R. Muller, D. Krusienski, G. Schalk, J. Wolpaw, A. Schlogl, G. Pfurtscheller, J. Millan, M. Schroder, and N. Birbaumer, "The BCI competition III: validating alternative approaches to actual BCI problems," Neural Systems and Rehabilitation Engineering, IEEE Transactions on, vol. 14, no. 2, pp. 153-159, 2006.

[4] A. Capilla, P. Pazo-Alvarez, A. Darriba, P. Campo, and J. Gross, "Steady-State Visual Evoked Potentials Can Be Explained by Temporal Superposition of Transient Event-Related Responses," PLOS ONE, vol. 6, no. 1, p. e14543, 2011. 

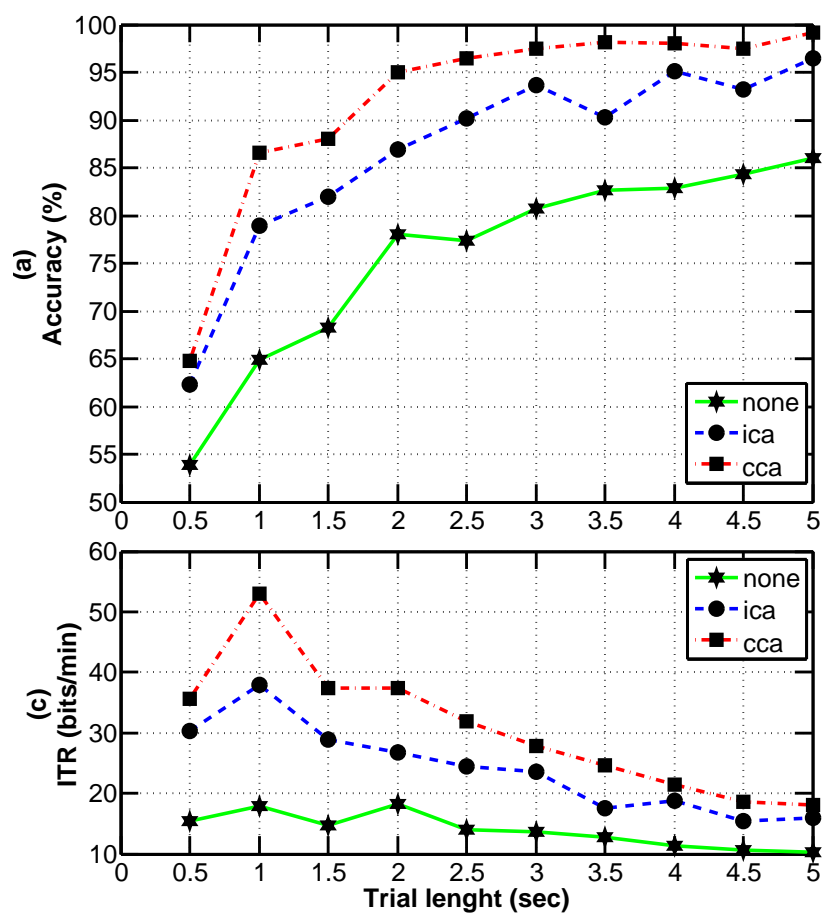

Fig. 5. A comparison of classification performances of an SVM classifier with (1) no signal enhancement, (2) ICA signal enhancement, and (3) CCA signal enhancement

[5] Y. Wang, Z. Zhang, X. Gao, and S. Gao, "Lead selection for SSVEPbased brain-computer interface," in Engineering in Medicine and Biology Society, 2004. IEMBS '04. 26th Annual International Conference of the IEEE, vol. 2, 2004, pp. 4507-4510.

[6] G. Pires, U. Nunes, and M. Castelo-Branco, "Statistical spatial filtering for a P300-based BCI: Tests in able-bodied, and patients with cerebral palsy and amyotrophic lateral sclerosis," Journal of Neuroscience Methods, vol. 195, no. 2, pp. 270-281, 2011.

[7] H. Lee and S. Choi, "PCA-based linear dynamical systems for multichannel EEG classification," in Neural Information Processing, 2002. ICONIP '02. Proceedings of the 9th International Conference on, vol. 2, 2002, pp. 745-749.

[8] C. Guan, M. Thulasidas, and J. Wu, "High performance P300 speller for brain-computer interface," in Biomedical Circuits and Systems, 2004 IEEE International Workshop on, 2004, pp. S3/5/INV - S3/13-16.

[9] D. R. Hardoon, S. R. Szedmak, and J. R. Shawe-Taylor, "Canonical Correlation Analysis: An Overview with Application to Learning Methods," Neural Comput., vol. 16, no. 12, pp. 2639-2664, 2004.

[10] Z. Lin, C. Zhang, W. Wu, and X. Gao, "Frequency Recognition Based on Canonical Correlation Analysis for SSVEP-Based BCIs," Biomedical Engineering, IEEE Transactions on, vol. 53, no. 12, pp. 2610-2614, 2006.

[11] G. Bin, X. Gao, Z. Yan, B. Hong, and S. Gao, "An online multi-channel SSVEP-based brain-computer interface using a canonical correlation analysis method," Journal of Neural Engineering, vol. 6, no. 4, 2009.

[12] D. Zhang, A. Maye, X. Gao, B. Hong, A. K. Engel, and S. Gao, "An independent brain-computer interface using covert non-spatial visual selective attention." Journal of neural engineering, vol. 7, no. 1, p. 16010, 2010.

[13] J. Pan, X. Gao, F. Duan, Z. Yan, and S. Gao, "Enhancing the classification accuracy of steady-state visual evoked potential-based brain-computer interfaces using phase constrained canonical correlation analysis," Journal of neural engineering, vol. 8, no. 3, p. 036027, 2011.

[14] Y. Renard, F. Lotte, G. Gibert, M. Congedo, E. Maby, V. Delannoy, O. Bertrand, and A. Lécuyer, "OpenViBE: An open-source software platform to design, test, and use brain-computer interfaces in real and virtual environments," Presence: Teleoperators and Virtual Environments, vol. 19, no. 1, pp. 35-53, 2010.
[15] C. C. Chang and C. J. Lin, "LIBSVM: A library for support vector machines," ACM Transactions on Intelligent Systems and Technology, vol. 2, no. 3, 2011.

[16] Y. Wang and T.-P. Jung, "Improving braincomputer interfaces using independent component analysis," in Towards Practical Brain-Computer Interfaces, 2013, pp. 67-83. 